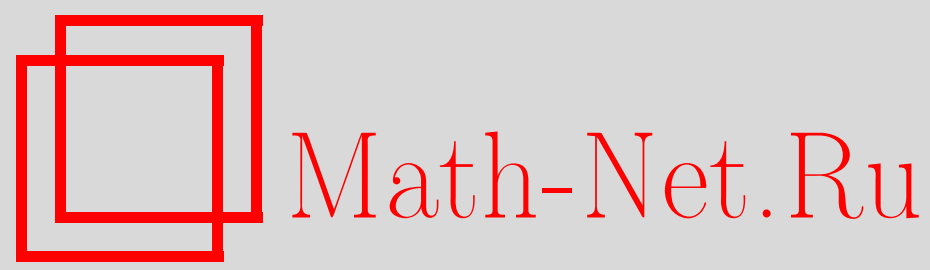

С. А. Теляковский, О равномерной сходимости тригонометрических рядов с редко меняющимися коэффициентами, Матем. заметки, 2001, том 70, выпуск 4, 613-620

DOI: https://doi.org/10.4213/mzm773

Использование Общероссийского математического портала Math-Net.Ru подразумевает, что вы прочитали и согласны с пользовательским соглашением http://www.mathnet.ru/rus/agreement

Параметры загрузки:

IP : 54.147 .182 .235

26 апреля 2023 г., 17:08:07

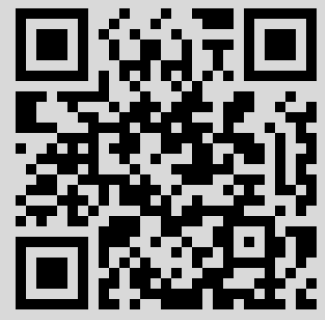




\section{О РАВНОМЕРНОЙ СХОДИМОСТИ ТРИГОНОМЕТРИЧЕСКИХ РЯДОВ \\ С РЕДКО МЕНЯЮЩИМИСЯ КОЭФФИЦИЕНТАМИ}

\section{С. А. Теляковский}

Рассматриваются ряды $\sum_{k=1}^{\infty} a_{k} \sin k x$ и $\frac{a_{0}}{2}+\sum_{k=1}^{\infty} a_{k} \cos k x$, коэффициенты которых удовлетворяют условию $a_{k}=a_{n_{m}}$ для $n_{m-1}<k \leqslant n_{m}$, где последовательность $\left\{n_{m}\right\}$ представима в виде объединения конечного числа лакунарных последовательностей. Получены следуюшие результаты. Если $k a_{k} \rightarrow 0$ при $k \rightarrow \infty$, то ряд $\sum_{k=1}^{\infty} a_{k} \sin k x$ сходится равномерно. Если $k\left|a_{k}\right| \leqslant C$ для всех $k$, то последовательность частных сумм этого ряда равномерно ограничена. Если ряд $\frac{a_{0}}{2}+\sum_{k=1}^{\infty} a_{k} \cos k x$ сходится при $x=0$ и $k a_{k} \rightarrow 0$ при $k \rightarrow \infty$, то этот ряд сходится равномерно. Если последовательность частных сумм ряда $\frac{a_{0}}{2}+\sum_{k=1}^{\infty} a_{k} \cos k x$ при $x=0$ ограничена и $k\left|a_{k}\right| \leqslant C$ для всех $k$, то последовательность частных сумм этого ряда равномерно ограничена. В этих утверждениях условия на скорость убывания коэффициентов рядов являются также необходимыми, если последовательность $\left\{n_{m}\right\}$ лакунарна. В общем случае они не являются необходимыми.

Библиографиял: 3 названия.

Известно (см. [1, с. 96, 98]), что если коэффициенты ряда по синусам

$$
\sum_{k=1}^{\infty} a_{k} \sin k x
$$

монотонно убьвают к нулю, то для равномерной сходимости этого ряда необходимо и достаточно выполнения условия $k a_{k} \rightarrow 0, k \rightarrow \infty$, а для равномерной ограниченности частных сумм ряда (1) необходимо и достаточно выполнения условия $k a_{k} \leqslant C$.

Здесь и в дальнейшем через $C$ обозначаются абсолютные положительные постоянные, в разных случаях различные.

В [2, теорема 3] приведенньй результат о равномерной сходимости распространен на ряды с квазимонотонными коэффициентами, когда для некоторого положительного числа $\alpha$ монотонно убывают числа $k^{-\alpha} a_{k}$.

В настоящей работе указьвается еще один класс рядов типа (1), для которых справедливы такие же утверждения, как для рядов с монотонными коэффициентами.

Работа выполнена при финансовой поддержке Российского фонда фундаментальных исследований, грант № 99-01-01210, и INTAS, грант № 99-01080. 
Поскольку в нашем случае коэффициенты $a_{k}$ не обязаны иметь одинаковые знаки, то такие же вопросы будут рассмотрены и для рядов по косинусам

$$
\frac{a_{0}}{2}+\sum_{k=1}^{\infty} a_{k} \cos k x
$$

Будем говорить, что последовательность $\left\{a_{k}\right\}$ удовлетворяет условию $\Delta_{L}$, если

$$
a_{k}=a_{n_{m}} \quad \text { для } n_{m-1}<k \leqslant n_{m}, \quad m=1,2, \ldots,
$$

где последовательность $\left\{n_{m}\right\}, n_{0}=0, n_{1}=1$, представима в виде объединения конечного числа лакунарных последовательностей. Таким образом, $\Delta a_{k}=a_{k}-a_{k+1}=0$ для всех $k \neq n_{m}, m=1,2, \ldots$

Известно [1, с. 24], [3, лемма 1]), что каждое из следующих двух условий является необходимьм и достаточным для того, чтобы последовательность $\left\{n_{m}\right\}$ можно было разбить на конечное число лакунарных последовательностей:

$$
\begin{aligned}
& \sum_{k=m}^{\infty} \frac{1}{n_{k}} \leqslant \frac{C}{n_{m}}, \quad m=1,2, \ldots, \\
& \sum_{k=1}^{m} n_{k} \leqslant C n_{m}, \quad m=1,2, \ldots .
\end{aligned}
$$

Теорема 1. Пусть коэффичиенты ряда (1) удовлетворяют условию $\Delta_{L}$.

Если $k a_{k} \rightarrow 0$ при $k \rightarrow \infty$, то ряд (1) сходится равномерно.

Если $k\left|a_{k}\right| \leqslant C$ для всех $k$, то последовательность частных сумм ряда (1) равномерно ограничена.

ДокАЗАТЕЛЬСТво. Запишем условия на скорость убьвания коэффициентов ряда (1) следующим образом: существует монотонно убьвающая последовательность $\{\alpha(k)\}$ такая, что $k\left|a_{k}\right| \leqslant \alpha(k)$. При этом если $k a_{k} \rightarrow 0$, то $\alpha(k) \downarrow 0$.

Так как

$$
\left|\Delta a_{n_{m}}\right| \leqslant\left|a_{n_{m}}\right|+\left|a_{n_{m+1}}\right| \leqslant \frac{2}{n_{m}} \alpha\left(n_{m}\right),
$$

TO

$$
\sum_{k=1}^{\infty}\left|\Delta a_{k}\right|=\sum_{m=1}^{\infty}\left|\Delta a_{n_{m}}\right| \leqslant \sum_{m=1}^{\infty} \frac{2}{n_{m}} \alpha\left(n_{m}\right) \leqslant 2 \alpha(1) \sum_{m=1}^{\infty} \frac{1}{n_{m}} .
$$

Последний ряд сходится в силу условия (4).

Отсюда следует, что ряд (1) сходится для всех $x$. Обозначим его сумму через $g(x)$. Докажем сначала утверждение теоремы о равномерной сходимости.

По заданному $n$ найдем число $p$ такое, что

$$
n_{p-1}<n \leqslant n_{p}
$$

Так как

$$
g(x)-s_{n-1}(g, x)=g(x)-s_{n_{p}-1}(g, x)+s_{n_{p}-1}(g, x)-s_{n-1}(g, x)
$$




$$
\left|s_{n_{p}-1}(g, x)-s_{n-1}(g, x)\right|=\left|\sum_{k=n}^{n_{p}-1} a_{k} \sin k x\right|=\left|a_{n_{p}}\right|\left|\sum_{k=n}^{n_{p}-1} \sin k x\right| \leqslant n_{p}\left|a_{n_{p}}\right| \leqslant \alpha\left(n_{p}\right),
$$

то достаточно оценить разности вида

$$
g(x)-s_{n_{p}-1}(g, x)
$$

При оценке этой разности будем пользоваться представлением

$$
g(x)-s_{n_{p}-1}(g, x)=\sum_{k=n_{p}}^{\infty} a_{k} \sin k x=\sum_{m=p}^{\infty} \Delta a_{n_{m}} \widetilde{D}_{n_{m}}(x)-a_{n_{p}} \widetilde{D}_{n_{p}-1}(x),
$$

где $\widetilde{D}_{k}(x)$ - сопряженное ядро Дирихле порядка $k$.

Поскольку

$$
\left|a_{n_{p}} \widetilde{D}_{n_{p}-1}(x)\right| \leqslant \alpha\left(n_{p}\right),
$$

задача сводится к оценке ряда

$$
\sum_{m=p}^{\infty} \Delta a_{n_{m}} \widetilde{D}_{n_{m}}(x)
$$

Для каждой точки $x \in(0, \pi]$ найдем число $j$ такое, что

$$
x \in\left(\frac{\pi}{n_{j+1}}, \frac{\pi}{n_{j}}\right] .
$$

Если вьполнено условие $(12)$ и $j<p$, то в силу (6) и (4) имеем

$$
\begin{aligned}
\left|\sum_{m=p}^{\infty} \Delta a_{n_{m}} \widetilde{D}_{n_{m}}(x)\right| & \leqslant \frac{C}{x} \sum_{m=p}^{\infty}\left|\Delta a_{n_{m}}\right| \leqslant C n_{j+1} \sum_{m=p}^{\infty} \frac{1}{n_{m}} \alpha\left(n_{m}\right) \\
& \leqslant C n_{j+1} \alpha\left(n_{p}\right) \sum_{m=p}^{\infty} \frac{1}{n_{m}} \leqslant C n_{j+1} \alpha\left(n_{p}\right) \frac{1}{n_{p}} \leqslant C \alpha\left(n_{p}\right) .
\end{aligned}
$$

Рассмотрим теперь случай, когда $j \geqslant p$. Запишем сумму из (11) в виде

$$
\sum_{m=p}^{\infty}=\sum_{m=p}^{j}+\sum_{m=j+1}^{\infty}
$$

Сумма по $m \geqslant j+1$ оценивается по аналогии с оценкой (13):

$$
\begin{aligned}
\left|\sum_{m=j+1}^{\infty} \Delta a_{n_{m}} \widetilde{D}_{n_{m}}(x)\right| & \leqslant \frac{C}{x} \sum_{m=j+1}^{\infty}\left|\Delta a_{n_{m}}\right| \\
& \leqslant C n_{j+1} \alpha\left(n_{j+1}\right) \sum_{m=j+1}^{\infty} \frac{1}{n_{m}} \leqslant C \alpha\left(n_{p}\right) .
\end{aligned}
$$


При оценке суммы по $m$ от $p$ до $j$ будем пользоваться неравенством

$$
\left|\widetilde{D}_{k}(x)\right|=\left|\sum_{i=1}^{k} \sin i x\right| \leqslant \sum_{i=1}^{k} i x \leqslant k^{2} x
$$

Согласно $(15),(12),(6)$ и (5) имеем

$$
\begin{aligned}
\left|\sum_{m=p}^{j} \Delta a_{n_{m}} \widetilde{D}_{n_{m}}(x)\right| & \leqslant x \sum_{m=p}^{j} n_{m}^{2}\left|\Delta a_{n_{m}}\right| \leqslant \frac{C}{n_{j}} \sum_{m=p}^{j} n_{m} \alpha\left(n_{m}\right) \\
& \leqslant \frac{C}{n_{j}} \alpha\left(n_{p}\right) \sum_{m=p}^{j} n_{m} \leqslant C \alpha\left(n_{p}\right) .
\end{aligned}
$$

Из (7)-(10), (13), (14) и (16) находим, что для всех $x$ и $n$ вьполнена оценка

$$
\left|g(x)-s_{n-1}(g, x)\right| \leqslant C \alpha(n) .
$$

Таким образом, установлено утверждение теоремы о равномерной сходимости ряда (1) и при этом получена оценка скорости сходимости (17).

Сходньги рассуждениями доказьвается и утверждение о равномерной ограниченности частных сумм.

Именно, с помощью оценки типа оценки (8) заключаем, что достаточно рассмотреть суммы вида $s_{n_{p}}(g, x)$.

Так как

$$
s_{n_{p}}(g, x)=\sum_{m=1}^{p} \Delta a_{n_{m}} \widetilde{D}_{n_{m}}(x)+a_{n_{p}+1} \widetilde{D}_{n_{p}}(x),
$$

то из условия $k\left|a_{k}\right| \leqslant C$ следует, что нужно получить оценку суммы

$$
\sum_{m=1}^{p} \Delta a_{n_{m}} \widetilde{D}_{n_{m}}(x)
$$

Если для $x$ вьполнено условие $(12)$ и $j \geqslant p$, то в силу оценок $(15),(6)$ и (5) имеем

$$
\left|\sum_{m=1}^{p} \Delta a_{n_{m}} \widetilde{D}_{n_{m}}(x)\right| \leqslant x \sum_{m=1}^{p} n_{m}^{2}\left|\Delta a_{n_{m}}\right| \leqslant \frac{C}{n_{j}} \alpha(1) \sum_{m=1}^{p} n_{m} \leqslant \frac{C}{n_{j}} \alpha(1) n_{p} \leqslant C \alpha(1) .
$$

А если $j<p$, то выделяем из суммы (18) слагаемые с $m \leqslant j$, для которых справедлива оценка (19), где нужно заменить $p$ на $j$. Оценка оставшихся слагаемых из (18) проводится по аналогии с (13):

$$
\left|\sum_{m=j+1}^{p} \Delta a_{n_{m}} \widetilde{D}_{n_{m}}(x)\right| \leqslant \frac{C}{x} \sum_{m=j+1}^{p}\left|\Delta a_{n_{m}}\right| \leqslant C n_{j+1} \alpha\left(n_{j+1}\right) \sum_{m=j+1}^{p} \frac{1}{n_{m}} \leqslant C \alpha\left(n_{j+1}\right) .
$$

Таким образом, при условии $k\left|a_{k}\right| \leqslant C$ частные суммы ряда (1) равномерно ограничены, и теорема полностью доказана. 
ТЕОРема 2. Пусть коэффициенты ряда (2) удовлетворяют условию $\Delta_{L}$.

Если ряд (2) сходится при $x=0 u k a_{k} \rightarrow 0$ прu $k \rightarrow \infty$, то ряд (2) сходится равномерно.

Если последовательность частных сумм ряда (2) ограничена при $x=0$ $u \quad k\left|a_{k}\right| \leqslant C$ для всех $k$, то последовательность частных сумм ряда (2) равномерно ограничена.

ДокАЗАТЕЛЬСтво. В силу оценки (6) ряд (2) сходится на $(0, \pi]$. Пусть $f(x)$ обозначает его сумму. Для простоты записей будем считать $a_{0}=0$. Понятно, что это не ограничивает общность.

Пусть величины $\alpha(k)$ имеют тот же смысл, что и в доказательстве теоремы 1 . Как и при доказательстве теоремы 1 , по $n$ находим число $p$, для которого вьполняются неравенства ( 7$)$, и убеждаемся, что при $x \in(0, \pi]$ справедливы оценки

$$
\left|f(x)-s_{n-1}(f, x)\right| \leqslant\left|\sum_{m=p}^{\infty} \Delta a_{n_{m}} D_{n_{m}}^{*}(x)\right|+C \alpha(n),
$$

где $D_{k}^{*}(x):=\sum_{i=1}^{k} \cos i x$, и

$$
\left|s_{n}(f, x)\right| \leqslant\left|\sum_{m=1}^{p} \Delta a_{n_{m}} D_{n_{m}}^{*}(x)\right|+C \alpha(n)
$$

когда ряд (2) сходится при $x=0$.

В случае сходимости ряда (2) наряду с $\alpha(k)$ введем монотонно убывающие к нулю величины $\beta(k)$ такие, что

$$
\left|\sum_{i=k}^{\infty} a_{i}\right| \leqslant \beta(k)
$$

В дальнейшем будет нужна оценка суммы

$$
\sum_{m=p}^{j} n_{m} \Delta a_{n_{m}}=\sum_{k=n_{p}}^{n_{j}} k \Delta a_{k}=\sum_{k=n_{p}}^{n_{j}} a_{k}+a_{n_{p}}\left(n_{p}-1\right)-a_{n_{j}+1} n_{j} .
$$

В силу этого представления имеем

$$
\left|\sum_{m=p}^{j} n_{m} \Delta a_{n_{m}}\right| \leqslant 2 \beta\left(n_{p}\right)+2 \alpha\left(n_{p}\right)
$$

Пусть $x$ удовлетворяет условию (12).

Тогда для $j<p$ по аналогии с (13) получим оценку

$$
\left|\sum_{m=p}^{\infty} \Delta a_{n_{m}} D_{n_{m}}^{*}(x)\right| \leqslant C \alpha\left(n_{p}\right)
$$


При $j \geqslant p$ точно так же устанавливается оценка

$$
\left|\sum_{m=j+1}^{\infty} \Delta a_{n_{m}} D_{n_{m}}^{*}(x)\right| \leqslant C \alpha\left(n_{p}\right) .
$$

Осталось рассмотреть сумму по $m$ от $p$ до $j$. В этом случае будем использовать оценку

$$
\left|D_{k}^{*}(x)-k\right|=\left|\sum_{i=1}^{k}(\cos i x-1)\right| \leqslant \sum_{i=1}^{k} i x \leqslant k^{2} x, \quad 0<x \leqslant \pi,
$$

аналогичную (15). Тогда с помощью (20), (21), (6), (12) и (5) находим

$$
\begin{aligned}
\left|\sum_{m=p}^{j} \Delta a_{n_{m}} D_{n_{m}}^{*}(x)\right| & \leqslant\left|\sum_{m=p}^{j} \Delta a_{n_{m}}\left(D_{n_{m}}^{*}(x)-n_{m}\right)\right|+C \beta\left(n_{p}\right)+C \alpha\left(n_{p}\right) \\
& \leqslant \sum_{m=p}^{j}\left|\Delta a_{n_{m}}\right| n_{m}^{2} x+C \beta\left(n_{p}\right)+C \alpha\left(n_{p}\right) \\
& \leqslant C x \sum_{m=p}^{j} \alpha\left(n_{m}\right) n_{m}+C \beta\left(n_{p}\right)+C \alpha\left(n_{p}\right) \leqslant C \alpha\left(n_{p}\right)+C \beta\left(n_{p}\right) .
\end{aligned}
$$

Собрав вместе полученные результаты, приходим к оценке

$$
\left|f(x)-s_{n-1}(f, x)\right| \leqslant C \alpha(n)+C \beta(n),
$$

доказьвающей утверждение теоремы 2 о равномерной сходимости и дающей, кроме того, оценку скорости сходимости.

Утверждение о равномерной ограниченности частных сумм также устанавливается по аналогии с доказательством соответствующей части теоремы 1 . Нужно будет только пользоваться оценкой (21) подобно тому, как это делалось вьше.

Понятно, что в теореме 2 условия на поведение ряда (2) при $x=0$ являются необходимыми. При этом легко убедиться, что они не вытекают из условий на скорость стремления к нулю коэффициентов $a_{k}$. Например, если $n_{m}:=2^{m}$ и $a_{2^{m}}:=1 /\left(m 2^{m}\right)$, то $k a_{k} \rightarrow 0$, а ряд $\sum a_{k}$ расходится.

В теоремах 1 и 2 условия $k a_{k} \rightarrow 0$ и $k\left|a_{k}\right| \leqslant C$ не являются в общем случае необходимыми.

В самом деле, последовательность $\left\{2^{m}-1,2^{m}\right\}, m=1,2, \ldots$, является объединением двух лакунарных последовательностей. Значит, последовательность коэффициентов

$$
a_{k}:= \begin{cases}m^{-2} & \text { для } k=2^{m}, \\ 0 & \text { для } k \neq 2^{m}\end{cases}
$$

удовлетворяет условию (3). Ряды (1) и (2) для этих коэффициентов сходятся равномерно, а вместе с тем условие $k\left|a_{k}\right| \leqslant C$ не вьполнено.

Но если последовательность $\left\{n_{m}\right\}$ в условии (3) является лакунарной, то в теоремах 1 и 2 условия на скорость стремления коэффициентов $a_{k}$ к нулю являются уже и необходимьми. 
Теорема 3. Пусть коэффициенты ряда (1) удовлетворяют условию (3) для лакунарной последовательности $\left\{n_{m}\right\}$. Тогда если ряд (1) равномерно сходится, mо $k a_{k} \rightarrow 0$ при $k \rightarrow \infty$, а если частные суммы ряда (1) равномерно ограничены, $m o k\left|a_{k}\right| \leqslant C$.

Такие же утверждения справедливы и для ряда (2).

ДокАЗАТЕЛЬСТво. Пусть

$$
\begin{aligned}
\frac{n_{m+1}}{n_{m}} & \geqslant q>1, \quad m=1,2, \ldots \\
n_{m}^{*} & :=\max \left(n_{m},\left[\frac{n_{m+1}}{2}\right]\right) .
\end{aligned}
$$

Тогда

$$
\frac{n_{m+1}}{n_{m}^{*}} \geqslant q^{*}:=\min (q, 2), \quad m=1,2, \ldots
$$

Пусть ряд (1) равномерно сходится и $g(x)$ обозначает его сумму. Введем величины

$$
\gamma(k):=\max _{i \geqslant k}\left\|g(x)-s_{i}(g, x)\right\|_{C} .
$$

Значит, $\gamma(k) \downarrow 0$ и

$$
\left\|s_{n_{m+1}}(g, x)-s_{n_{m}^{*}}(g, x)\right\|_{C} \leqslant 2 \gamma\left(n_{m}\right)
$$

Ho

$$
s_{n_{m+1}}(g, x)-s_{n_{m}^{*}}(g, x)=a_{n_{m+1}} \sum_{k=n_{m}^{*}+1}^{n_{m+1}} \sin k x .
$$

Положив здесь $x=\pi /\left(2 n_{m+1}\right)$, видим, что

$$
\begin{aligned}
\left\|s_{n_{m+1}}(g, x)-s_{n_{m}^{*}}(g, x)\right\|_{C} & \geqslant\left|a_{n_{m+1}}\right| \sum_{k=n_{m}^{*}+1}^{n_{m+1}} \sin \frac{k \pi}{2 n_{m+1}} \geqslant\left|a_{n_{m+1}}\right| \sum_{k=n_{m}^{*}+1}^{n_{m+1}} \frac{k}{n_{m+1}} \\
& \geqslant\left|a_{n_{m+1}}\right| \frac{n_{m}^{*}+1}{n_{m+1}}\left(n_{m+1}-n_{m}^{*}\right) \\
& =n_{m+1}\left|a_{n_{m+1}}\right| \frac{n_{m}^{*}+1}{n_{m+1}}\left(1-\frac{n_{m}^{*}}{n_{m+1}}\right) .
\end{aligned}
$$

В силу определения чисел $n_{m}^{*}$ и неравенства (22) имеем

$$
\frac{n_{m}^{*}+1}{n_{m+1}}\left(1-\frac{n_{m}^{*}}{n_{m+1}}\right) \geqslant \frac{1}{2}\left(1-\frac{1}{q^{*}}\right)
$$

Таким образом, из (23)-(25) получаем

$$
2 \gamma\left(n_{m}\right) \geqslant n_{m+1}\left|a_{n_{m+1}}\right| \cdot \frac{1}{2}\left(1-\frac{1}{q^{*}}\right),
$$

откуда следует, что $k a_{k} \rightarrow 0$ при $k \rightarrow \infty$. 
Если же частные суммы ряда (1) равномерно ограничены, то величины

$$
a_{n_{m+1}} \sum_{k=n_{m}^{*}+1}^{n_{m+1}} \sin k x
$$

равномерно ограничены. Отсюда в силу проведенных оценок вытекает неравенство $k\left|a_{k}\right| \leqslant C$.

Для ряда (2) рассуждения аналогичны, но значения разностей

$$
s_{n_{m+1}}(g, x)-s_{n_{m}^{*}}(g, x)
$$

берутся при $x=0$. Тогда получаем

$$
\left\|s_{n_{m+1}}(f, x)-s_{n_{m}^{*}}(f, x)\right\|_{C}=\left|a_{n_{m+1}}\right|\left(n_{m+1}-n_{m}^{*}\right) \geqslant n_{m+1}\left|a_{n_{m+1}}\right|\left(1-\frac{1}{q^{*}}\right) .
$$

Отсюда вытекают оба утверждения теоремы 3 о ряде (2).

\section{СПИСОК ЦИТИРОВАННОЙ ЛИТЕРАТУРЫ}

[1] Бари Н. К. Тригонометрические ряды. М.: Физматгиз, 1961.

[2] Shah S.M. Trigonometric series with quasi-monotone coefficients // Proc. Amer. Math. Soc. 1962. V. 13. P. 266-273.

[3] Стечкин С. Б. Об абсолютной сходимости рядов Фурье (третье сообщение) // Изв. АН СССР. Сер. матем. 1956. Т. 20. С. 385-412. 